\title{
A Proteoliposome Containing Apolipoprotein A-I Mutant (V156K) Enhances Rapid Tumor Regression Activity of Human Origin Oncolytic Adenovirus in Tumor-Bearing Zebrafish and Mice
}

\author{
Juyi Seo ${ }^{1,2}$, Chae-Ok Yun ${ }^{3}$, Oh-Joon Kwon ${ }^{3}$, Eun-Jin Choi $^{4}$, Jae-Young Song ${ }^{4}$, Inho Choi ${ }^{1,2}$, and \\ Kyung-Hyun Cho ${ }^{1,2, *}$
}

\begin{abstract}
We recently reported that the efficiency of adenoviral gene delivery and virus stability are significantly enhanced when a proteoliposome (PL) containing apolipoprotein (apo) A-I is used in an animal model. In the current study, we tested tumor removal activity of oncolytic adenovirus (Ad) using PL-containing wildtype (WT) or V156K. Oncolytic Ad with or without PL was injected into tumors of zebrafish and nude mice as a Hep3B tumor xenograft model. The V156KPL-Ad-injected zebrafish, group showed the lowest tumor tissue volume and nucleic acids in the tumor area, whereas injection of Ad alone did not result in adequate removal of tumor activity. Reactive oxygen species (ROS) contents increased two-fold in tumor-bearing zebrafish; however, the V156K-PL-Ad injected group showed a $40 \%$ decrease in ROS levels compared to that in normal zebrafish. After reducing the tumor volume with the V156K-PLAd injection, the swimming pattern of the zebrafish changed to be more active and energetic. The oncolytic effect of PLAd containing either V156K or WT was about two-fold more enhanced in mice than that of Ad alone $\mathbf{3 4}$ days after the injection. Immunohistochemical analysis revealed that the PL-Ad-injected groups showed enhanced efficiency of viral delivery with elevated Ad-E1A staining and a diminished number of proliferating tumor cells. Thus, the antitumor effect of oncolytic Ad was strongly enhanced by a PL-containing apoA-I and its mutant (V156K) without causing side effects in mice and zebrafish models.
\end{abstract}

\section{INTRODUCTION}

Viruses are widely used tools for therapeutic gene delivery of many critical diseases. Although a few hurdles have been overcome to achieve practical success, one promising group of anticancer agents is the oncolytic viruses (Cattaneo et al., 2008).
Among oncolytic viruses, oncolytic adenovirus (Ad) is a widely used anti-tumor activity tool for many different tumor types (Toth et al., 2010). A number of preclinical and clinical studies have used oncolytic Ad with encouraging results (Kumar et al., 2008). Of note, an in vivo evaluation of an adenoviral gene delivery was limited due to the lack of a suitable animal model. Infection with Ad is species-specific, and the replication ability of human-originating $\mathrm{Ad}$ is much lower than that in laboratory animals. Development of a new experimental animal model is needed prior to testing larger animals and conducting clinical trials. Zebrafish (Danio rerio) are a good model to test gene delivery efficiency because a zebrafish homologue of the coxsackievirus and Ad receptor protein has been identified (Petrella et al., 2002). Zebrafish can serve as a chemical treatment cancer model or through transplantation of mammalian cells (Feitsma and Cuppen, 2008).

Ad has immunogenic properties and induces inflammatory activity (Yang et al., 1994). Additionally, the stability and efficiency of Ad is weakened at increased temperatures. To overcome these disadvantages, development of an Ad vehicle may help protect the stability of Ad. We recently reported that the efficiency of gene delivery is significantly enhanced using a proteoliposome $(\mathrm{PL})$ containing apolipoprotein $A-I$ in various cell and zebrafish models (Park et al., 2010). We also showed that human-originating Ad replicates in zebrafish. Adult zebrafish can be infected with Ad, particularly with PL containing apoA-I. A PL containing V156K (V156K-PL-Ad) has near twofold enhanced GFP expression than a PL containing a wildtype apoA-I (WT-PL-Ad), raising the possibility that Ad can be formulated in $\mathrm{PL}$ as a novel tool for targeted gene delivery, which would have a variety of therapeutic applications (Park et al., 2010). The formulation of Ad in PL greatly enhances the efficiency of adenoviral gene delivery in various cell and zebrafish models. Additionally, the anti-inflammatory and antioxidant activities of apoA-I administered in this fashion could be used to

${ }^{1}$ School of Biotechnology, Yeungnam University, Gyeongsan 712-749, Korea, ${ }^{2}$ Research Institute of Protein Sensor, Yeungnam University, Gyeongsan 712-749, Korea, ${ }^{3}$ Department of Bioengineering, College of Engineering, Hanyang University, Seoul 133-791, Korea, ${ }^{4}$ Viral Disease Division, Animal, Plant and Fisheries Quarantine and Inspection Agency (QIA), Anyang 430-757, Korea,

${ }^{*}$ Correspondence: chok@yu.ac.kr 
treat cancer.

In the current study, we assessed the tumor removal effect of oncolytic Ad alone or Ad in WT-PL-Ad or V156K-PL-Ad using tumor-bearing zebrafish.

\section{MATERIALS AND METHODS}

\section{Materials}

Dimyristoyl phosphatidylcholine (DMPC; \#121518, $1 \mathrm{~g}$ ) and cholesterol (\# C8667) were purchased from Avanti Polar Lipids, Inc. (USA) and Sigma (USA), respectively.

\section{Purification of WT and V156K apoA-I}

WT and V156K apoA-I were expressed in an E. coli expression system and then purified using $\mathrm{Ni}^{2+}$-column chromatography as described previously (Cho et al., 2006; Han et al., 2005). Protein purity was assessed by $20 \%$ sodium dodecyl sulfate-poly acrylamide gel electrophoresis using a Pharmacia Phast System (Uppsala, Sweden). The functional and structural characterization of the apoA-I in lipid-free state and lipid-bound state was carried out according to our previous report (Cho, 2009a; Park and Cho, 2011)

\section{Generation of oncolytic Ad regulated by the recombinant HRE-AFP promoter}

To construct oncolytic Ads targeting hepatocellular carcinoma, the recombinant AFP promoter excised from pBlueScriptll $(+)$ was sub-cloned into phAFP-d19, pre-digested with Sall/Xhol, and the six copies of HRE, which were digested with Sall/Xhol from pTeasy/HRE $\mathrm{H}_{6}$, were inserted into an E1 shuttle vector containing the modified AFP promoter. The resulting shuttle vector (pHa2bmd19) was linearized with $X m n l$, then co-transformed into E. coli BJ5183 together with the BstBI-digested adenoviral plasmid (vmdl324BstBI) for homologous recombination.

The oncolytic Ad (Ha2bm-d19) was propagated in 293A cells, purified by the cesium chloride density purification method, dissolved in storage solution (10 $\mathrm{mM}$ Tris, $4 \%$ sucrose, and 2 $\mathrm{mM} \mathrm{MgCl} 2$ ), and stored at $-80^{\circ} \mathrm{C}$ before use according to a standard protocol (Hitt et al., 1994). Viral particle numbers were calculated from measurements of optical density at $260 \mathrm{~nm}$, in which 1 absorbance unit was equivalent to $1 \times 10^{12}$ viral particles per $\mathrm{ml}$.

\section{Synthesis of a PL with oncolytic Ad}

PL containing DMPC, free cholesterol (FC), and apoA-I were synthesized by sonication using an initial molar ratio of DMPC: FC: apoA-I of $200: 9: 1$. During synthesis, $10 \mu$ of the oncolytic Ad stock solution (final concentration, $1.2 \times 10^{9}$ particles $/ \mu$ l of $\mathrm{PL}$ ) was added to the PL, which was incubated at room temperature for $24 \mathrm{~h}$ to allow association of the PL and Ad, as described previously (Park et al., 2010).

\section{Zebrafish and tumor induction}

Zebrafish and zebrafish embryos were maintained according to standard protocols (Nusslein-Volhard, 2002). Procedures involving zebrafish were approved by the Committee of Animal Care and Use of Yeungnam University (Korea). The zebrafish larvae and embryos were maintained in a system cage and 6well plates at $28^{\circ} \mathrm{C}$ during treatment under a $14: 10 \mathrm{~h}$ light: dark cycle. Tetrabit were used as the basal diet, supplemented with Atremia salina nauplii.

Primary tumors were induced in 11-week-old zebrafish by continuous immersion in $\mathrm{N}$-nitrosodiethylamine (DEN) solution
(100 ppm) for 8 weeks, as described previously (Mizgireuv and Revskoy, 2006). Thirty zebrafish were maintained in a 10-L glass tank equipped with a mechanical filter and automatic heater during the DEN exposure. After the exposure, the fish were transferred to a 20-L tank and were observed for up to 9 months.

\section{Zebrafish microinjection}

WT-PL-Ad or V156K-PL-Ad were individually microinjected using a pneumatic picopump (PV820; World Precision Instruments, USA) equipped with a magnetic manipulator (MM33; Kantec, USA) with a pulled microcapillary pipette-using device (PC-10; Narishigen, Japan) into 36-week-old zebrafish, which had grossly visible neoplasms located on the ventral region. Injections were performed at the same site on the tumor with a total of $100 \mathrm{nl}$ of V156K-PL-Ad or WT-PL-Ad $\left(5.7 \times 10^{6}\right.$ virus particle, $36 \mathrm{ng}$ of apoA-I) to minimize bias as previously reported by Cho group (Park and Cho, 2011). Filter-sterilized solutions with the same number of Ad in each vehicle were injected into zebrafish after anesthetization with tricaine methane sulfonate (Sigma A5040, final concentration, 0.002\%).

After injection, live zebrafish were observed under a stereomicroscope (Motic SMZ 168, Hong Kong, China) and photographed using a motic cam 2300 CCD camera.

\section{Histological analysis}

After three injections per day within a 24-h interval for up to 72 hours, all zebrafish were sacrificed, fixed in $4 \%$ solution of paraformaldehyde, and microsectioned with a cryotome (MICROM, HM500; Germany). The tumor site in the ventral region was sectioned longitudinally from head-to-tail and stained with hematoxyin and eosin. In order to compare extent of DNA replication in the tumor site, we employed fluorescent dye using DAPI as previously described (Kapuscinski, 1990). Photographs of the slides were obtained using a Nikon Eclipse TE2000 microscope (Japan) at $600 \times$ magnification.

\section{Reactive oxygen species (ROS) imaging}

After treatment of zebrafish with Ad or PL-Ad, the increased ROS and inflammatory response in the injected area were imaged using dihydroethidium (DHE, Sigma cat \# 37291) as described previously (Owusu-Ansah et al., 2008). The stained image of tumor site was obtained by fluorescence observation $(E x=588 \mathrm{~nm}$ and $\mathrm{Em}=605 \mathrm{~nm}$ ) using a Nikon Eclipse TE2000 microscope. Quantification of the fluorescence area in the injected area was carried out via computer-assisted morphometry using Image Proplus software (version 4.5.1.22; Media Cybernetics, USA).

\section{Anti-tumor effects of Ad-PL complexes in the liver cancer xenograft model}

To establish the Hep3B tumor xenograft model, $1 \times 10^{7}$ cells were subcutaneously injected into the abdomens of 7-week-old male athymic nu/nu mice (Orientbio Inc, Korea). When tumor volume reached about $150 \mathrm{~mm}^{3}$, the mice were divided into four separate groups ( $n=6$ per group) and injected intratumorally with $30 \mu \mathrm{lPBS}$ or $3 \times 10^{9}$ VP of Ha2bm-d19, Ha2bmd19:V156K, or Ha2bm-d19:WT in $30 \mu \mathrm{l}$ of PBS three times every other day. Tumor growth was measured with calipers every 2 days, and tumor volume was calculated (volume $=0.523 \times$ length $\times$ width $^{2}$ ). 


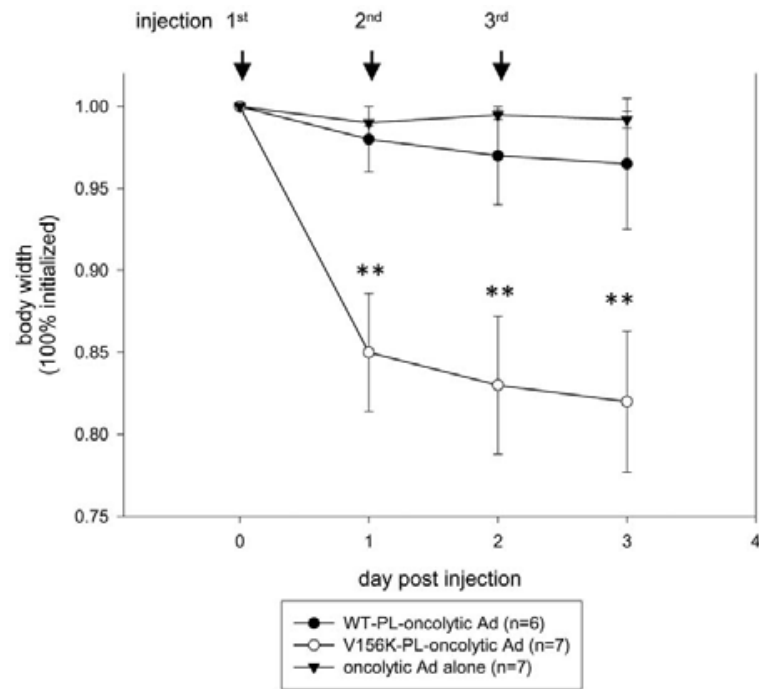

Fig. 1. Tumor-removal effect due to injection of a proteoliposomeadenovirus (PL-Ad) or Ad alone. The change in the longest body width of the tumor area was measured by stereomicroscopy at a designated time.

\section{Evaluation of tumor xenografts by histology and immunohistochemistry}

Hep3B tumors that were treated with PBS, Ha2bm-d19, Ha2bmd19:V156K, or Ha2bm-d19:WT were collected at 3 days after the final injection and fixed in $10 \%$ formalin, embedded in paraffin (Wax-it, Canada), and sectioned at $5 \mu \mathrm{m}$. Representative sections were stained with hematoxylin and eosin and for adenoviral E1A protein or proliferating cell nuclear antigen (PCNA) by incubating the sections with rabbit anti-Ad E1A (sc-430; Santa Cruz Biotechnology, USA) or mouse anti-PCNA (M0879, Dakocytomation, USA).

\section{RESULTS AND DISCUSSION}

\section{Tumor removal by V156K-PL-oncolytic Ad}

Exposure to water-soluble carcinogens, such as DEN, can induce a wide array of benign and malignant tumors in many different organs (Spitsbergen et al., 2000). Although it is unclear what kind of cancer occurs following DEN treatment, zebrafish can serve as a chemical treatment cancer model or by transplantation of mammalian cells (Mizgireuv and Revskoy, 2006).

Twenty-three weeks after DEN immersion and at the same age (36 week-old), the control and DEN-treated zebrafish groups had weight-to-height $(\mathrm{mg} / \mathrm{mm})$ ratios of 14.5 and 11.1 , respectively. Initially, both groups had a weight-to-height ratio of 15.1, as shown in Supplementary Fig. 1A. But, the DEN-treated zebrafish had a $20 \%$ reduction in body weight after 23 weeks, whereas the control group had a $6 \%$ increase in body weight. The DEN group had a $5 \%$ increase in height, whereas the control group had a $13 \%$ increase in height. These results suggest that weight loss occurred as a function of tumorigenesis. The grossly visible tumors, approximately $2-7 \mathrm{~mm}$ in diameter, appeared in the abdominal area 14-23 weeks after exposure to DEN as indicated by the arrow (Supplementary Fig. 1B).

The three daily injections (24 h interval) of Ad in PL (100 nl, $5.7 \times 10^{6} \mathrm{VP}$ ) into solid tumor sites of DEN-treated or control zebrafish were well-tolerated. Only one tumor-bearing zebrafish died after the first injection of WT-PL-Ad. The tumor area in the ventral region decreased significantly $24 \mathrm{~h}$ following the V156KPL-Ad injection [up to a $15 \%$ decrease in body width from the initial value (from $4.5 \mathrm{~mm}$ before injection to $3.9 \mathrm{~mm}$ )] (Fig. 1). The decrease was detected 3 days post-injection (up to an $18 \%$ decrease in body width) as shown in Fig. 1 and Supplementary Figs. 2 and 3. However, WT-PL-Ad-injected zebrafish only showed a $2-4 \%$ decrease in body width from the initial value during the 3 days. The Ad alone-injected group had almost no change in body width or tumor size. The same injection of each PL-Ad into either DEN-treated or control zebrafish did not result in any toxicity or swimming defects. The DENtreated zebrafish (Supplementary Video 1) with a tumor in the ventral region exhibited a slower and weaker swimming pattern. However, $24 \mathrm{~h}$ after injection (Supplementary Video 2), the swimming pattern of the V156K-PL-Ad-injected zebrafish was more active compared to that prior to injection, as the tumor had disappeared.

\section{Histological analysis}

The tumor site in the ventral area was micro-sectioned longitudinally from head-to-tail and stained with hematoxylin and eosin. As shown in Fig. 2, a larger number of hematoxylin-stained cells was detected at the tumor site of DEN-treated zebrafish compared to that in control zebrafish. In addition, tumor sections showed less eosin-staining efficiency than normal tissue, suggesting that rapidly dividing cells, such as cancer cells, are less prone to staining, as reported by Le et al. (2007). The DHE staining to visualize ROS showed that normal muscle exhibited a stronger red intensity due to eosin staining and fewer nuclei compared with those in tumor tissue, which had a larger number nuclei of increased size with weaker red staining intensity (Fig. 3).

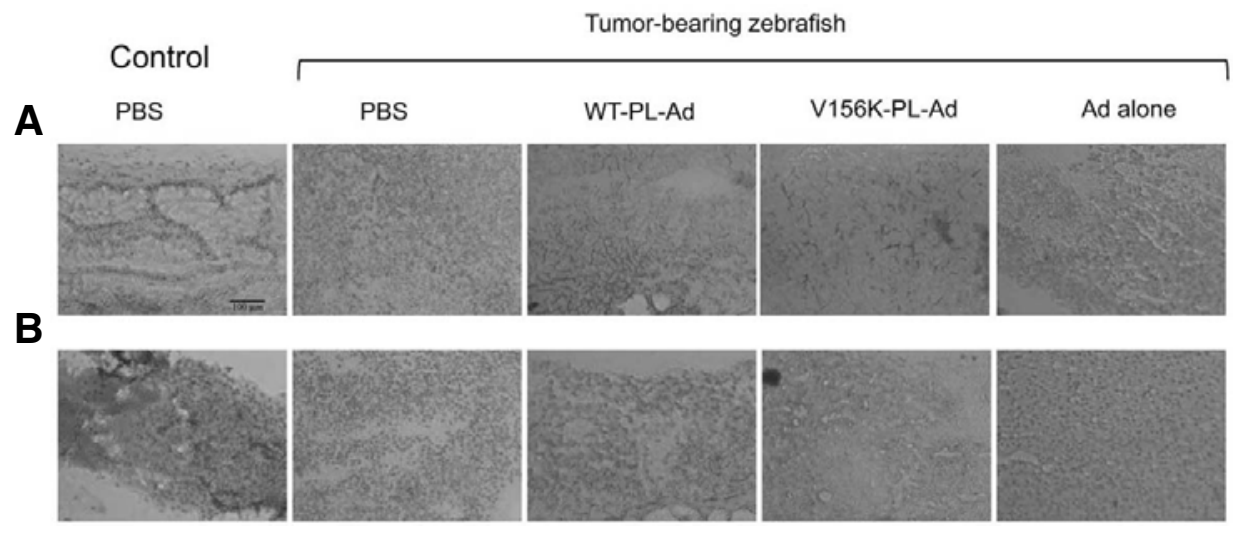

Fig. 2. Longitudinal section $(7 \mu \mathrm{m}$ thickness) from head-to-tail in the ventral region, including the tumor was sectioned and stained with hematoxylin and eosin ( $\mathrm{H} \& \mathrm{E}) 3$ days post-injection. Representative section at $420 \mu \mathrm{m}$ depth from the surface $(A)$ and at $800 \mu \mathrm{m}$ depth (B) $600 \times$ magnification. 
A

Normal-zebrafish PBS

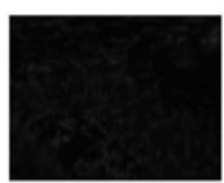

$\mathrm{DHE}$

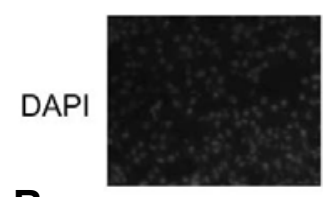

B

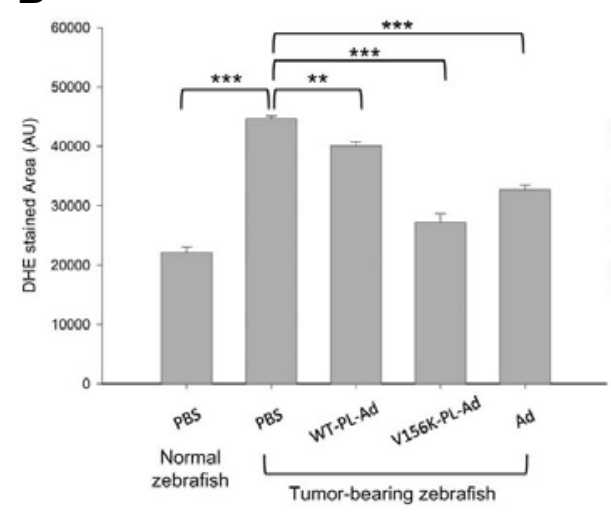

DHE staining revealed that the ROS level increased in the tumor induced area of the DEN treated group, suggesting that rapid tumorigenesis was accompanied by an acute inflammatory response. This result was in good agreement with a previous report in a DEN-treated rat model, which showed oxidative stress and an inflammatory response due to DEN-initiated hepatocarcinoma (Bishayee et al., 2010). Among tumor bearing zebrafish, the V156K-PL-Ad injected group showed the lowest intensity of DHE- and DAPI-stained cells, whereas the PBSinjected group showed the highest intensity.

We recently reported that rapamycin, a non-soluble drug, becomes more solubilized in saline solution when reconstituted in high-density lipoproteins (HDL) containing V156K-apoA-I (V156KrHDL). Rapamycin in V156K-rHDL shows enhanced delivery efficiency and an anti-inflammatory effect in the zebrafish model (Cho, 2011). Furthermore, V156K-rHDL exhibits anti-inflammatory activity in apo-E deficient mice and is more resistant to myeloperoxidase-mediated oxidation (Cho and Kim, 2009; Cho et al., 2007).

More recently, we showed that V156K-rHDL has potent antioxidant activity and is more resistant to glycation with enhanced tissue regeneration activity in the zebrafish model (Yoon and Cho, 2012). Increased resistance to glycation is advantageous to develop protein-based drugs as anti-atherosclerotic and antidiabetic agents. These previous reports that showed enhanced properties of V156K-apoA-I in PL or rHDL were in good agreement with the current tumor-removal results in the zebrafish model.

The V156K-PL-Ad-injected group had a decreased hematoxylin-stained area than that observed in the WT-PL-Ad-or Ad alone-injected zebrafish, indicating that the number and size of nuclei was diminished by eliminating the tumor. These results
Fig. 3. Detection of the amount of reactive oxygen species (ROS) by DHE staining (Ex $=588 \mathrm{~nm}$, Em = $605 \mathrm{~nm}$ ) and the extent of doublestranded nucleic acid replication by DAPI staining (Ex $=350 \mathrm{~nm}$, $\mathrm{Em}=460 \mathrm{~nm}$ ). (A) Representative fluorescence image of microsectioned tumor tissue. (B) Quantification of fluorescence in the microsectioned slides using computer-assisted morphometry based on the same size $\left(7.4 \mathrm{~mm}^{2}\right)$ as the DHE and DAPI staining. Data are mean \pm standard deviation from three independent experiments performed in duplicate. ${ }^{*} p<0.05$; ${ }^{* *} p<0.01 ;{ }^{* * *} p<0.001$.

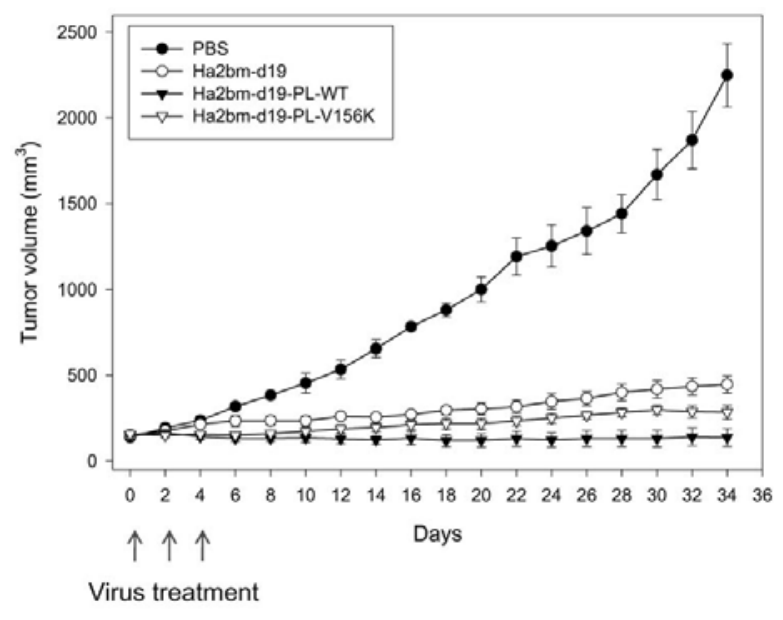

Fig. 4. Intratumoral injection of Ad alone or PL-Ad into Balb/c nude mice, which was established as the Hep3B tumor xenograft model. The same amount of $\mathrm{Ad}\left(3 \times 10^{9}\right.$ virus particles) was injected three times as indicated by the arrow, and tumor volume was measured at 34 days post-injection.

correlated well with the stereoscopic microscope observations. Indeed, the disappearance of the tumor in the ventral region was associated with a decrease in hematoxylin-stained cells in V156K-PL-Ad-injected zebrafish.

Anti-tumor effect in the Hep3B subcutaneous model Tumor volume in PBS-injected Hep3B tumor xenograft model 


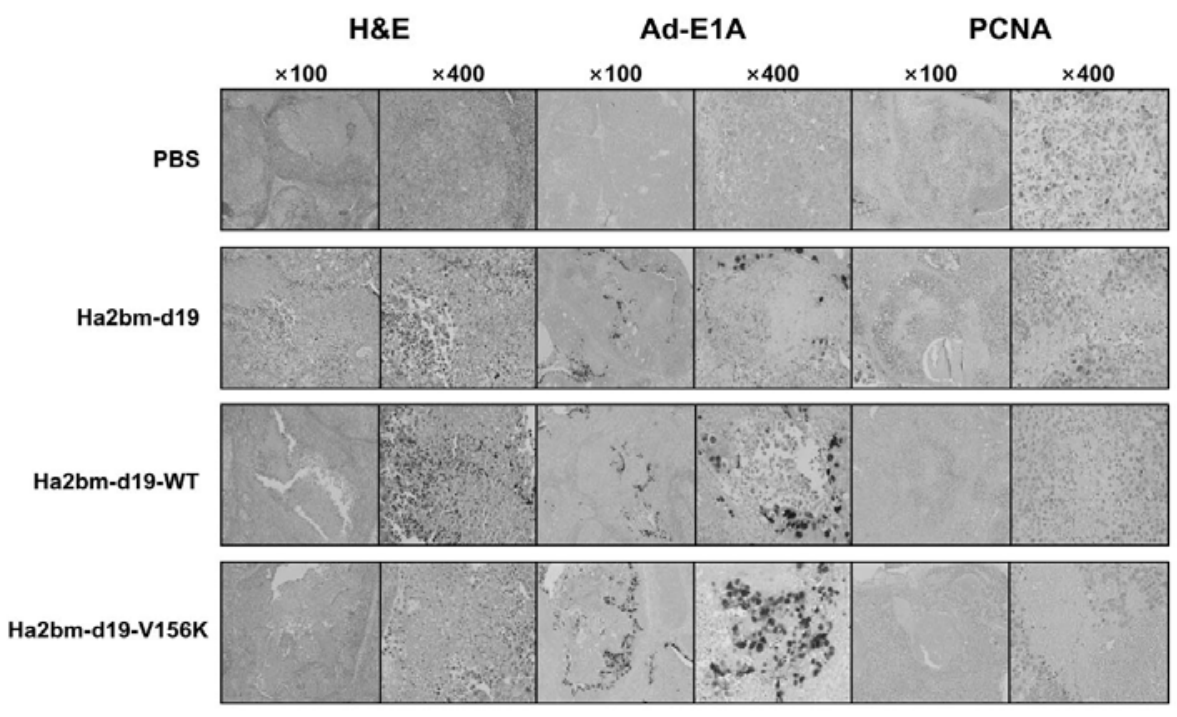

Fig. 5. Histological analysis of tumor area at 3 days post final injection by hematoxylin and eosin $(H$ \& E) staining. Particles of adenovirus (Ad) in the tumor area were visualized by immunohistochemistry using the antiAd E1A antibody. Proliferating cell nuclear antigen (PCNA) was visualized by immunodetection using mouse antiPCNA antibody.

mice during 34 days increased to $2248 \pm 185 \mathrm{~mm}^{3}$, whereas that in the oncolytic Ad (Ha2bm-d19) injected group had a tumor volume of $446 \pm 50 \mathrm{~mm}^{3}$. This result suggests that the speed of tumor growth could be attenuated by up to $80 \%$ by injecting oncolytic Ad. Furthermore, the PL-Ad injected group showed much less tumor growth in both V156K and WT (284 \pm 41 and $137 \pm 52 \mathrm{~mm}^{3}$, respectively) (Fig. 4). The PL-Ad injected group showed only $8 \%$ and $6 \%$ of tumor volume compared with that in the PBS-control, indicating that the oncolytic effect of $\mathrm{Ad}$ was greatly enhanced by the PL formulation. The immunohistochemical analysis revealed that PL-Ad containing either V156K or WT showed enhanced viral delivery (Ad-E1A staining) and diminished proliferating tumor cell (PCNA staining) (Fig. 5).

These results suggest that the PL-Ad containing either WT or V156K showed potent inhibitory activity against tumor growth, which was xenografted in the mice. In contrast with these results, the tumor eliminating activity of WT-PL-Ad in the zebrafish was much weaker, suggesting that there might be speciesspecific tumorigenesis mechanisms. Although it is unclear why WT-PL-Ad showed a different efficacy between the zebrafish and mice models, it is likely that the two cancer models have a different tumorigenic mechanism. The tumor in the zebrafish model was a spontaneous tumor caused by a chemical carcinogen, whereas the tumor in nude mice was established by transplantation of tumor cells. Future research should be conducted to address the oncolytic effect of tumorigenesis in different models. Another possibility is that the WT-apoA-I was more sensitive to the protein modification by oxidative stress as our previous report (Park and Cho, 2011; Yoon and Cho, 2012). Generally, apoA-I exerts antioxidant and anti-inflammatory activity in human and animal plasma (Kim et al., 2010; Patel et al., 2010) and anti-tumor activity in mouse ovarian cancer (Su et al., 2010). Due to many beneficial effects of apoA-I in plasma, infusion of HDL/apoA-I therapy has been well established to treat atherosclerosis and coronary artery disease (Cho, 2009b; Tardif, 2009).

Preclinical anti-tumor experiments are usually conducted in immune-deficient mice (nude and SCID) or a hamster model (Thomas et al., 2006), which is more expensive and time consuming. As we have demonstrated in the current report, zebraf- ish are a good cancer model to evaluate putative tumor regression activity with a low maintenance cost. This is the first study to show enhanced delivery efficiency of Ad, particularly the tumor eliminating activity of oncolytic Ad. Although the oncolytic Ad has been used to treat cancer (Toth and Wold, 2010), it is necessary to develop more enhanced delivery methods for better efficacy.

In conclusion, the current results demonstrated that epidermal injection of human-origin apoA-I-PL-oncolytic Ad eliminated carcinogen-induced tumors in zebrafish without causing side effects. As with oncolytic Ad, other viruses for gene therapy can be formulated with V156K-PL. This V156K-PL-Ad system can be applied to humans and other vertebrates for further efficacy testing of virotherapy.

Note: Supplementary information is available on the Molecules and Cells website (www.molcells.org).

\section{ACKNOWLEDGMENTS}

This work was supported by the Mid-carrier Researcher Program (2011-0015529), through the National Research Foundation (NRF) of Korea by the Ministry of Education, Science and Technology (MEST) and supported by a grant from the Ministry for Food, Agriculture, Forestry and Fisheries, Republic of Korea to the Animal, Plant and Fisheries Quarantine and Inspection Agency (Project number: Z-AD14-2011-11-01).

\section{REFERENCES}

Bishayee, A., Barnes, K.F., Bhatia, D., Darvesh, A.S., and Carroll, R.T. (2010). Resveratrol suppresses oxidative stress and inflammatory response in diethylnitrosamine-initiated rat hepatocarcinogenesis. Cancer Prev. Res. 3, 753-763.

Cattaneo, R., Miest, T., Shashkova, E.V., and Barry, M.A. (2008). Reprogrammed viruses as cancer therapeutics: targeted, armed and shielded. Nat. Rev. Microbiol. 6, 529-540.

Cho, K.H. (2009a). Synthesis of reconstituted high-density lipoprotein (rHDL) containing apoA-I and apoC-III: the functional role of apoC-III in rHDL. Mol. Cells 27, 291-297

Cho, K.H. (2009b). Biomedicinal implications of high-density lipoprotein: its composition, structure, functions, and clinical applications. BMB Rep. 42, 393-400.

Cho, K.H. (2011). Enhanced delivery of rapamycin by V156K-apoA- 
I high-density lipoprotein inhibits cellular pro-atherogenic effects and senescence and promotes tissue regeneration. J. Gerontol. A. Biol. Sci. Med. Sci. 66, 1274-1285

Cho, K.H., and Kim, J.R. (2009). A reconstituted high-density lipoprotein containing V156K or R173C apoA-I exhibited antiinflammatory activity in apo-E deficient mice and showed resistance to myeloperoxidase-mediated oxidation. Exp. Mol. Med. 41, 417-428.

Cho, K.H., Park, S.H., Han, J.M., Kim, H.C., Choi, Y.K., and Choi, I. (2006). ApoA-I mutants V156K and R173C promote antiinflammatory function and antioxidant activities. Eur. J. Clin. Invest. 36, 875-882.

Cho, K.H., Park, S.H., Han, J.M., Kim, H.C., Chung, Y.J., Choi, I, and Kim, J.R. (2007). A point mutant of apolipoprotein A-I, V156K, exhibited potent anti-oxidant and anti-atherosclerotic activity in hypercholesterolemic C57BL/6 mice. Exp. Mol. Med. 39, 160-169.

Feitsma, H., and Cuppen, E. (2008). Zebrafish as a cancer model. Mol. Cancer Res. 6, 685-694.

Han, J.M., Jeong, T.S., Lee, W.S., Choi, I., and Cho, K.H. (2005). Structural and functional properties of V156K and A158E mutants of apolipoprotein A-I in the lipid-free and lipid-bound states. J. Lipid Res. 46, 589-596.

Hitt, M., Bett, A.J., Prevec, L., and Graham, F.L. (1994). Construction and propagation of human adenovirus vectors. Cell biology: a laboratory handbook. (Academic Press Inc. New York), pp. 479-490.

Kapuscinski, J. (1990). Interactions of nucleic acids with fluorescent dyes: spectral properties of condensed complexes. J. Histochem. Cytochem. 38, 1323-1329.

Kim, J., Park, H.H., Choi, I., Kim, Y.O., and Cho, K.H. (2010) Severely modified lipoprotein properties without a change in cholesteryl ester transfer protein activity in patients with acute rena failure secondary to Hantaan virus infection. BMB Rep. 43, 535540

Kumar, S., Gao, L., Yeagy, B., and Reid, T. (2008). Virus combinations and chemotherapy for the treatment of human cancers. Curr. Opin. Mol. Ther. 10, 371-379.

Le, X., Langenau, D.M., Keefe, M.D., Kutok, J.L., Neuberg, D.S., and Zon, Z.L. (2007). Heat shock-inducible Cre/Lox approaches to induce diverse types of tumors and hyperplasia in transgenic zebrafish. Proc. Natl. Acad. Sci. USA 104, 9410-9415.

Mizgireuv, I.V., and Revskoy, S.Y. (2006). Transplantable tumor lines generated in clonal zebrafish. Cancer Res. 66, 3120-3125.

Nusslein-Volhard, C., and Dahm, R. (2002). Zebrafish: A Practical Approach (Oxford University Press)

Owusu-Ansah, E., Yavari, A., Mandal, S., and Banerjee, U. (2008). Distinct mitochondrial retrograde signals control the G1-S cell cycle checkpoint. Nat. Genet. 4, 356-361.

Park, K.H., and Cho, K.H. (2011). A zebrafish model for the rapid evaluation of pro-oxidative and inflammatory death by lipopolysaccharide, oxidized low-density lipoproteins, and glycated high-density lipoproteins. Fish Shellfish Immunol. 31, 904-910.

Park, K.H., Yun, C.O., Kwon, O.J., Kim, C.H., Kim, J.R., and Cho, K.H. (2010). Enhanced delivery of an adenovirus using proteoliposomes containing WT or V156K apolipoprotein A-I and dimyristoylphosphatidylcholine. Hum. Gene Ther. 21, 579-587.

Patel, S., Di Bartolo, B.A., Nakhla, S., Heather, A.K., Mitchell, T.W Jessup, W., Celermajer, D.S., Barter, P.J., and Rye, K.A. (2010) Anti-inflammatory effects of apolipoprotein A-I in the rabbit. Atherosclerosis 212, 392-397.

Petrella, J., Cohen, C.J., Gaetz, J., and Bergelson, J.M. (2002). A zebrafish coxsackievirus and adenovirus receptor homologue interacts with coxsackie B virus and adenovirus. J. Virol. 76, 10503-10506.

Spitsbergen, J.M., Tsai, H.W., Reddy, A., Miller, T., Arbogast, D. Hendricks, J.D., and Bailey, G.S. (2000). Neoplasia in zebrafish (Danio rerio) treated with $\mathrm{N}$-methyl- $\mathrm{N}^{\prime}$-nitro-N-nitrosoguanidine by three exposure routes at different developmental stages. Toxicol. Pathol. 28, 716-725.

Su, F., Kozak, K.R., Imaizumi, S., Gao, F., Amneus, M.W., Grijalva V., Ng, C., Wagner, A., Hough, G., Farias-Eisner, G., et al. (2010). Apolipoprotein A-I (apoA-I) and apoA-I mimetic peptides inhibit tumor development in a mouse model of ovarian cancer. Proc. Natl. Acad. Sci. USA 107, 19997-20002.

Tardif, J.C., Heinonen, T., and Noble, S. (2009). High-density lipoprotein/apolipoprotein A-I infusion therapy. Curr. Atheroscler. Rep. 11, 58-63.

Thomas, M.A., Spencer, J.F., La Regina, M.C., Dhar, D., Tollefson, A.E., Toth, K., and Wold, W.S. (2006). Syrian hamster as a permissive immunocompetent animal model for the study of oncolytic adenovirus vectors. Cancer Res. 66, 1270-1276.

Toth, K., and Wold, W.S. (2010). Increasing the efficacy of oncolytic adenovirus vectors. Viruses 2, 1844-1866.

Toth, K., Dhar, D., and Wold, W.S. (2010). Oncolytic (replicationcompetent) adenoviruses as anticancer agents. Exp. Opin. Biol. Ther. 10, 353-368.

Yang, Y., Nunes, F.A., Berencsi, K., Furth, E.E., Gönczöl, E., and Wilson, J.M. (1994). Cellular immunity to viral antigens limits E1-deleted adenoviruses for gene therapy. Proc. Natl. Acad. Sci. USA 91, 4407-4411.

Yoon, J.H., and Cho, K.H. (2012). A point mutant of apolipoprotein A-I (V156K) showed enhancement of cellular insulin secretion and potent activity of facultative regeneration in zebrafish. Rejuvenation Res. 15, 313-321. 